\title{
ARTE COMUNITARIO: COHESIÓN ENTRE ARTE, ECOLOGÍA Y EDUCACIÓN
}

\author{
Ana María Marques Ibáñez \\ Universidad de La Laguna, Dpto. Bellas Artes
}

\section{Resumen}

Actualmente existen prácticas artísticas educativas donde coexisten nexos de unión con el arte contemporáneo y se destinan a proyectos sociales, con nociones propias del posmodernismo de reapropiación o intervención de los espacios periféricos, urbanos o naturales. Se manifiesta una correspondencia entre diversas concepciones como: cultura, arte y medio ambiente mediante experiencias artísticas interdisciplinares con el fin de estudiar cómo los artistas, comisarios, investigadores, fotógrafos, publicistas y arquitectos, exponen temas heterogéneos para solucionar conflictos a nivel local y proyectarlo como una idea global con una mirada desde la comunidad. Los aspectos a desarrollar más significativos son problemáticas sociales, de identidad cultural, calentamiento global, sequía, catástrofes naturales que ocasionan la destrucción del hábitat y la degradación del entorno. Es enriquecedor crear prácticas artísticas innovadoras generadoras de nuevas narrativas alternativas para el fomento del bienestar social. Se analiza cómo desde la cultura visual, el arte contemporáneo y la educación artística se interrelacionan ecología, sociología, antropología y justicia para aplicarlo al consumo racional de recursos.

\section{Palabras clave: ARTE COMUNITARIO; ECOLOGÍA; ARTE CONTEMPORÁNEO; CULTURA VISUAL; PRÁCTICAS ARTÍSTICAS COLABORATIVAS}

\section{COMMUNITY ART: COHESION BETWEEN ART, ECOLOGY AND EDUCATION}

\section{Abstract}

Today, a number of educational art practices are connected with contemporary art while being aimed at social projects. These practices are based on notions of postmodernism related to the reappropriation or intervention of peripheral urban and natural spaces. This paper presents the relationship between different concepts such as culture, art, and the environment, by means of interdisciplinary art experiences. The aim is to explore how artists, curators, researchers, photojournalists, publicists, and architects present heterogeneous themes to solve conflicts at the local level, and to present our findings as a global idea from the perspective of the community. The principal areas of interests include social problems, cultural identity, global warming, drought, natural disasters leading to the destruction of habitats, and environmental degradation. It is essential to create innovative and productive art projects that generate new and alternative narratives to promote social wellbeing. We analyse the relationship between ecology, sociology, anthropology, and justice within the fields of visual culture, contemporary art, and art education, and how these connections can be applied to the rational use of resources.

Keywords: COMMUNITY ART; ECOLOGY; CONTEMPORARY ART; VISUAL CULTURE; COLLABORATIVE ARTISTIC PRACTICES

\footnotetext{
Marques Ibáñez, Ana María. "Arte comunitario: Cohesión entre arte, ecología y educación". AusArt 6 (1): 95-114. D0I: 10.1387/ ausart.19466
}

\section{AUSART}




\section{INTRODUCCIÓN}

Se analizan algunos ejemplos de prácticas artísticas comprometidas socialmente para generar una mejora en la calidad de vida de una comunidad. Se examina el desarrollo que ha experimentado la historia del arte comunitario, su avance y se exponen actuaciones innovadoras en este ámbito. Investigación de diferentes términos relacionados con este arte y cómo se potencian a través de la práctica artística.

El arte participativo y comprometido socialmente establece un diálogo con el arte contemporáneo del que forma parte y de la cultura visual. Se estudian los géneros más importantes que influyen en el arte comunitario y cómo constituyen nuevas propuestas en arte. Así como, determinados artistas que han generado prácticas colaborativas fundamentadas en su comunidad con piezas que muestran claramente esta idea. La exposición de los diversos géneros de arte comunitario ofrece una reflexión sobre propuestas en arte de carácter sostenible, para crear un cambio positivo en el entorno.

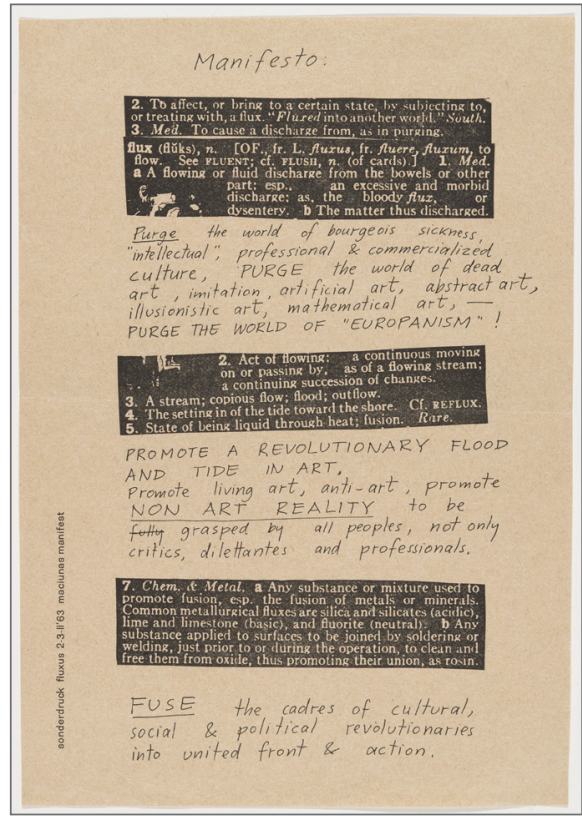

Fig. 1. Manifiesto Fluxus, 1963. George Maciunas https://www.moma.org/collection/works/127947 [Acceso: 10 feb 2018].

\section{CONCEPCIÓN DE LA CULTURA VISUAL CONTEMPORÁNEA}

Las imágenes de la cultura popular tienen una amplia repercusión en el arte, sobre todo las que proceden de la iconografía publicitaria. Esta idea se expuso en el manifiesto de la exposición High-Low, 1992 del Instituto de Arte de Chicago. Warhol y Lichtenstein rompen los límites establecidos de la representación de lo socialmente aceptado y lo muestran como popular. El grupo Fluxus unía las bellas artes con la cultura popular, como apareció en una exposición realizada en los centros de Arte Walker de Minneapolis y en el Wexner de Columbia. Los lenguajes del panorama artístico y la cultura visual contemporánea, precisan conocer la estructura sobre los que se construyeron los movimientos moderno y postmoderno. 


\section{REVISIÓN SOBRE LA DENOMINACIÓN DE ARTE COMUNITARIO}

El arte comunitario se construye mediante la correspondencia sobre procesos artísticos creativos orientados a una comunidad, así como a su entorno, por ello es fundamental examinar nuestro espacio de hábitat próximo. Los trabajos en arte comunitario presentan una interacción espacial o física y son prácticas generadoras de comunicación entre los miembros del grupo, y si existe la implicación de un artista se facilita el proceso en relación a una coordinación comprometida social y artísticamente. Estas propuestas comenzaron tras la Segunda Guerra Mundial como introducción del concepto democracia cultural, utilizado para nombrar actividades artísticas y culturales creadas por grupos y personas y no por instituciones.

En el año 1960 se creó un movimiento artístico que comienza en Estados Unidos y que se extendió todo el mundo y que daba protagonismo a colectivos sin visibilidad. Sus intervenciones se realizan en espacios degradados, los miembros de la comunidad local se agrupan para mejorar las condiciones de vida del entorno y sobre todo para canalizar sus problemas y darles visibilidad, las piezas artísticas son catalizadoras de ideas que generan acontecimientos y producen cambios en ámbitos locales o internacionales.

En los años 70 como expone Haacke $(1971,21)$ en una entrevista ofrecida Jeanne Siegel a la revista Arts Magazine se empezó a tomar conciencia sobre el valor de la denominación del arte conceptual, que posibilitaba cambios no sólo de origen estético, sino también que podían afectar a la mejora de una comunidad. "La información que se presenta en el lugar adecuado en el momento justo puede tener un gran poder. Puede afectar al tejido social [...]. La premisa básica es pensar en términos de sistemas: en la producción de sistemas, en descubrir y dificultar los sistemas existentes [...]. Los sistemas pueden ser físicos, biológicos o sociales". Como paradigma, el colectivo artístico Assemble con sede en Londres, ha creado proyectos de arte y arquitectura para comunidades y su calidad ha sido reconocida con el premio Turner en 2015'.

\subsection{Denominaciones artísticas con similitudes al arte COMUNITARIO}

El autor Owen Kelly, en su controvertida obra Community Art and the State: Storming the Citadels, comenta explícitamente el rol de este movimiento 
en la actividad política: "El concepto de arte comunitario no se refiere a ninguna actividad en específico o grupo de actividades, más bien define un acercamiento a la actividad creativa adoptando varios tipos de eventos y un amplio rango de medios. [...] El acercamiento utilizado en arte comunitario une a los artistas y a la gente local dentro de sus varias comunidades para usar formas de arte como un medio de comunicación y expresión, de un modo critico en el que usa y desarrolla artes de forma tradicionales, adaptándolas al presente desarrollando nuevos formatos [...] El arte comunitario propone el uso del arte para efectuar un cambio social y modificar las políticas sociales y engloba la expresión de acción política [...] Los activistas de arte comunitario operan en áreas deprimidas usando el término "privación" para incluir privación financiera, cultural, ambiental o educativa (Kelly 1984, 1-2) El término arte comunitario se emplea para los proyectos artísticos creados en una comunidad, pero se dan otras denominaciones que se asocian a éste, como: giro social y practica socialmente comprometida.

\section{Giro social}

Empleado para proyectos artísticos colaborativos comprometidos socialmente, que implican a personas a través de un medio específico o material de trabajo utilizado. el término fue creado por la historiadora de arte Claire Bishop, en su ensayo The Social Turn: Collaboration and Its Discontents, 2006. Entre las finalidades expuestas se sitúa la creación de arte colaborativo que promueva el cambio social, que ocasionalmente se muestra como comercial mediante objetos o su reproducción de forma seriada. Un ejemplo de pieza artística de giro social puede ser la de Tennantspin 1999, Superflex que crearon una televisión a través de internet para hogares de personas de edad avanzada en Liverpool.

\section{Práctica socialmente comprometida}

Arte participativo y colaborativo que implica a individuos mediante el entorno, en el que se establecen nexos de unión y diálogo, a través de interacción social. Se inician con programas de educación o divulgación, aunque también lo han realizado artistas independientes en su obra. La denominación fue creada por Suzanne Lacy para prácticas artísticas sociales, participativas e integradas en una comunidad para establecer diálogo, educar o compartir ideas. La finalidad es crear un proyecto para mostrar problemáticas, generar conciencia social y mejorar sus condiciones de vida. 


\subsection{GÉneros artísticos del arte comunitario}

El arte comunitario vincula la interacción creada en una comunidad y el arte, los individuos que la constituyen y habitan, el proyecto artístico basado en ideas de justicia aplicadas a lo social y las metodologías empleadas para educación. El arte destinado a una comunidad facilita un cambio en la sociedad y motiva a los individuos que la constituyen a realizar creaciones con profesionales del ámbito artístico. Estos procesos de creación artística, se refieren al ámbito local, regional nacional e internacional. En la actualidad el arte se involucra con la comunidad, con acciones sostenibles y medioambientales, como en proyectos de regeneración de zonas urbanas donde se establecen, plantean e implementan piezas artísticas en el barrio.

\section{Escultura ambiental}

Piezas escultóricas diseñadas que pretenden cambiar el entorno de sus habitantes, no es una representación figurativa y suelen ser de grandes dimensiones. En estas esculturas se permite el acceso a ellas. Su creación trata de producir sombras o reflejos, que modifican el color y la luz de una zona natural, urbana o periférica. La conocida comisaria Lippard (2004) expone en su libro esencial Seis años: La desmaterialización del objeto artístico de 1966 a 1972, proporciona las claves de los discursos y evolución de las piezas artísticas en este caso de arte ambiental que es nómada y efímero: Con el arte ambiental [...] ves al tiempo que te mueves y te mueves al tiempo que ves. El arte se hace transitorio, no cinético [...]. La historia es la conciencia del desarrollo de todo lo que ha habido antes de nosotros. Si podemos extraer de la historia la conciencia del proceso/desarrollo de nuestras vidas, podemos avanzar y ampliar nuestra propia conciencia [...]. Se puede concebir que, conforme se haga innecesario aferrarse a las cosas y con la ventaja de no emplear en nuestras mentes como recipientes de almacenamiento, la humanidad puede alcanzar un nivel de conciencia nunca conseguido antes (Lippard 2004, 124). Posteriormente Eberhard Bosslet desde 1983 efectúa intervenciones en ruinas, tituladas como reconstrucciones modificadas a través de efectos secundarios en referencia a la actuación sobre edificios de tipo industrial y áreas residenciales, dibujando líneas blancas o áreas de color negras. Elena Paroucheva diseñó en 1999 una intervención para torres de electricidad con piezas escultóricas. 


\section{Instalación artística}

Son obras tridimensionales pensadas para ser ubicadas en un sitio específico y modificar la percepción previa que teníamos de ese espacio. En general esta noción es utilizada para espacios interiores, debido a que las instalaciones en exterior reciben la denominación de arte público, arte de intervención o arte de la tierra, aunque estas nociones se muestran superpuestas y no existen unos límites establecidos.

\section{Arte público}

Arte creado, planificado y ejecutado con la finalidad de difundirlo en los medios a un público amplio y situado en un área accesible y abierta. Esta modalidad artística ha adquirido importancia en la historia del arte, entre los profesionales que difunden las nociones de arte contemporáneo, los comisarios y artistas. Para éstos últimos se valora y analiza el sitio específico, la colaboración de la comunidad y su participación activa. Este discurso comienza a través de la relación entre la audiencia y el contenido expuesto.

\section{Arte participativo}

Es una modalidad de arte en la que el proceso creativo se genera con la participación de los que habitualmente son espectadores pasivos y su relación a veces es visitar o adquirir obras de arte. El arte participativo tiene antecedentes en muchos movimientos y culturas, pero en su concepción actual citamos a Allan Kaproov conocido por sus happenings.

\section{Arte colaborativo}

Se inicia en los años sesenta con ideas ligadas a reivindicaciones políticas y se asocia al incremento de performances. Empleado por colectivos feministas, ecologistas y activismo de grupos con problemática específica y con un interés marcado en incluir el arte en su contexto pasando a expresarse fuera de las galerías e instituciones y saliendo a expresar sus piezas en espacios públicos, es por esto que se denomina como arte público. Se centra en facilitar la difusión de una problemática para facilitar su resolución. Fomenta visibilidad a colectivos y facilita su integración mediante la puesta en valor del grupo y de sus integrantes. Principalmente al dotarles de medios de expresión y facilitar su participación en la sociedad con un mayor conocimiento y valoración de su identidad y así participar en los procesos de mejora social. 


\section{Arte relacional}

La estética de este género artístico ha sido analizada por Nicolás Bourriaud, que lo definió como: grupo de prácticas en arte que parten de un formato teórico - práctico fomentando las relaciones humanas, así como sucontexto social, en vez de propiciar un lugar privado e independiente. El creador en el arte relacional es catalizador del proceso no su protagonista.

\subsection{Formatos narrativos emergentes en arte comunitario}

Las nuevas formas de narración en arte comunitario se construyen en prácticas colaborativas con formatos diferentes. En estos modelos en arte existe un compromiso e interrelación con una comunidad específica, se fomentan mediante prácticas colaborativas para compartir conocimientos en arte y se edifican en torno a la justicia social entre personas sensibilizadas con las desigualdades.

En el formato que se basa en la figura del artista, siendo el creador el protagonista, para visualizar lo que ha sido obviado y propiciar cambios sociales, mediante el impacto de sus obras. Por ejemplo, cuando pinta murales muestra la problemática de una comunidad. En otros casos, los artistas activan en ella la creación de obras, la difusión de ideas y la adquisición de una conciencia crítica. En la tercera tipología, los creadores realizan con un grupo el proceso creativo como en el circo social o a través de la danza, con fines terapéuticos; dando protagonismo al grupo o planificando obras para ser expuestas.

Estos modelos son específicos de su comunidad y sus prácticas colaborativas se modifican a través de la acción conjunta. Por esto, antes de visualizar los discursos o narrativas generados se deben analizar los modelos en los que se basan, estos prototipos se crean desde la participación directa o mediante comunidades virtuales, interviniendo grupos de diferentes disciplinas. El arte es creado para construir un cambio social, una democracia fundamentada en la cultura que facilita la participación. Las acciones comunitarias se inician a través de temas educativos, valores de las comunidades como los indígenas o inmigrantes y en visibilizar la problemática de los jóvenes. 


\subsection{Artistas relevantes y proyectos específicos de arte COMUNITARIO}

Existen una serie de artistas y proyectos específicos que son claves para el análisis del arte comunitario, desde sus inicios como vertiente artística, así como para su continuación y desarrollo. En la actualidad, se ha revalorizado este tipo de arte a través del Aprendizaje basado en Proyectos artísticos con un fin social y en el que se implica la comunidad.

Seguidamente se describen artistas canarios vinculados a este movimiento artístico social, pues las prácticas artísticas deben estar enraizadas y con sentido de identidad del espacio donde van a realizarse.

\section{Joseph Beuys}

Participo desde sus inicios en el arte comunitario, artista perteneciente al grupo Fluxus colaboró activamente mediante performances, instalaciones y esculturas, además de trabajar como pedagogo y teórico del arte. Desarrolla significados innovadores como la noción extendida del arte, la escultura social como una pieza de arte total (denominación creada por el compositor Richard Wagner, en relación a una tipología artística en la que se fusiona: música, poesía danza, pintura, arquitectura y escultura). Participó en política desde su faceta de artista con debates sobre la sociedad y su implicación en temas sociales y medioambientales. Una de sus más conocidas intervenciones fue una propuesta de plantar 7000 robles en 1982 en la Documenta 7 de Kassel, durante un periodo de varios años con ayuda de voluntarios y que finalizó cinco años después, en la Documenta 8. Cada uno de los robles estaba acompañado por una piedra de basalto. Beuys lo nombró escultura social, fue el trabajo de una comunidad para la intervención de un espacio de un
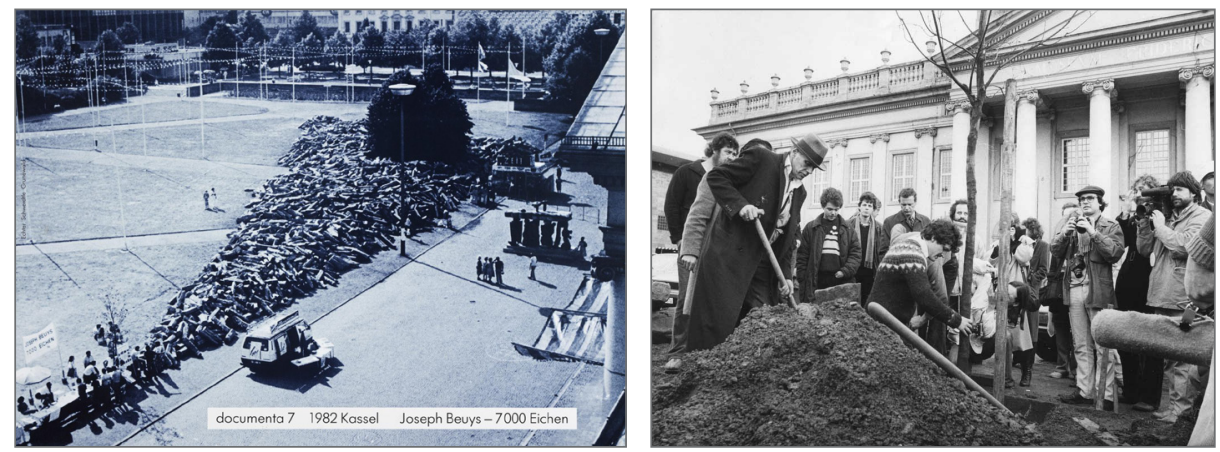

Fig. 2 y 3. 7000 Oaks: City Forestation Instead of City Administration (1982-1987. Joseph Beuys. www.tate.org.uk/art/artworks/beuys-7000-oak-trees-ar00745/ http://kassel.de/kultur/documenta/kunstwerke/objete/08259/index.html [Acceso 8 oct. 2017] 
modo físico, metafórico y espiritual; que posteriormente tomaría el nombre de ecourbanización.

\section{Vik Muniz}

Artista nacido en São Paulo de reconocido prestigio que se caracteriza por su experimentación con materiales diversos y fue a través de su película de carácter documental: WasteLand, 2010, cuando realizó un viaje al lugar donde nació para documentar y fotografiar a los trabajadores del vertedero más grande del mundo: Jardín Gramacho. El artista diseñó unos autorretratos de grandes dimensiones. La finalidad del proyecto era mostrar a los trabajadores una visión renovada sobre su identidad y su comunidad, que se encuentran en zonas marginales y condiciones peligrosas en el trabajo. La venta de las fotografías realizadas a los retratos obtiene recursos para los trabajadores y mejorar sus condiciones de vida.

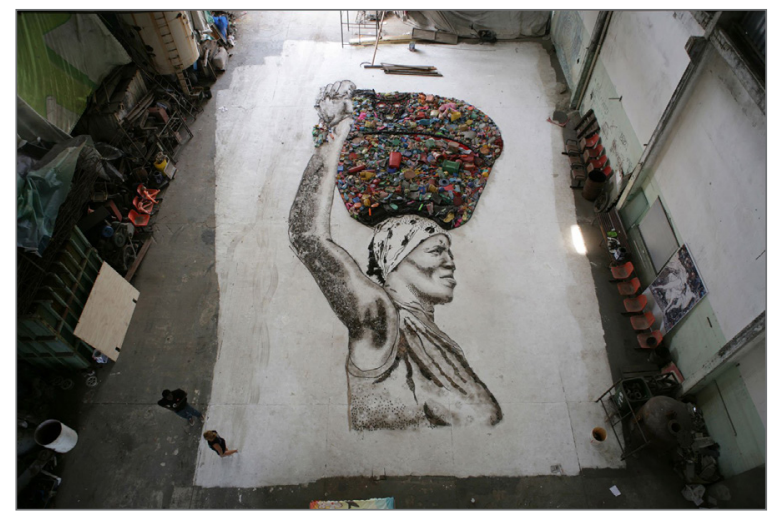

Fig. 4. Retrato de Irma. Trabajadora de Jardín Gramacho, 2009. Portraits with Purpose: Vik Muniz in Waste Land. Sikkema Jenkins y Co. http://time. com/3775724/portraits-with-purpose-vik-muniz-in-waste-land/ . [Acceso 15 feb. 2017]

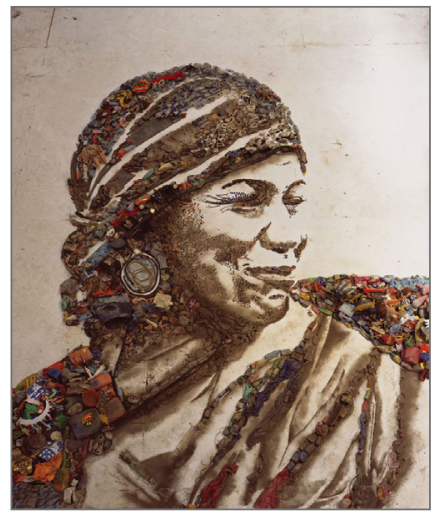

Fig. 5. The Gypsy Magna, de la serie Fotos de basura. Vik Muniz-Sikkema Jenkins y Co. Documental: Wasteland. Director: Lucy Walker. http://time.com/3775724/portraitswith-purpose-vik-muniz-in-wasteland/ [Acceso 15 feb. 2017]

\section{Basurama}

Colectivo formado por arquitectos, diseñadores y profesionales relacionados con el arte que a través de proyectos sociales fomentan un nuevo contexto para mejorar el entorno. Como el orfanato estatal de Kibebe Tsehay en Addis Abeba donde residen menores de los 0 a 8 años de edad. El exterior del centro tenía un aspecto descuidado y el mobiliario de juego deteriorado acumulándose también objetos inservibles. El proyecto se basa en la metáfora 
los Niños Perdidos del cuento de James Matthew Barrie, y fomenta un espacio donde crear privacidad para los niños y la realización de un patio donde jugar de forma segura. La intervención se ejecutó en diez días, en varias fases: búsqueda de recursos y materiales, diseño, coordinación, y realización; intervinieron voluntarios de la Universidad de Addis Abeba y del Instituto Cervantes, así como personas que acudieron a la llamada realizada en una radio local. Se construyó con palés de madera en los que se identificaban las ramas de los árboles con los que habían sido fabricados, restos metálicos de los columpios y mobiliario existente. Estos materiales se emplearon para la construcción de una estructura de un tubo hecho de metal, que aseguraba una durabilidad de la intervención. La instalación del espacio lúdico se completó con una cubierta creada para que los participantes se extendieran en la zona realizada con una lona publicitaria. Se pintaron en rojo objetos encontrados de los niños del orfanato como recuerdo.
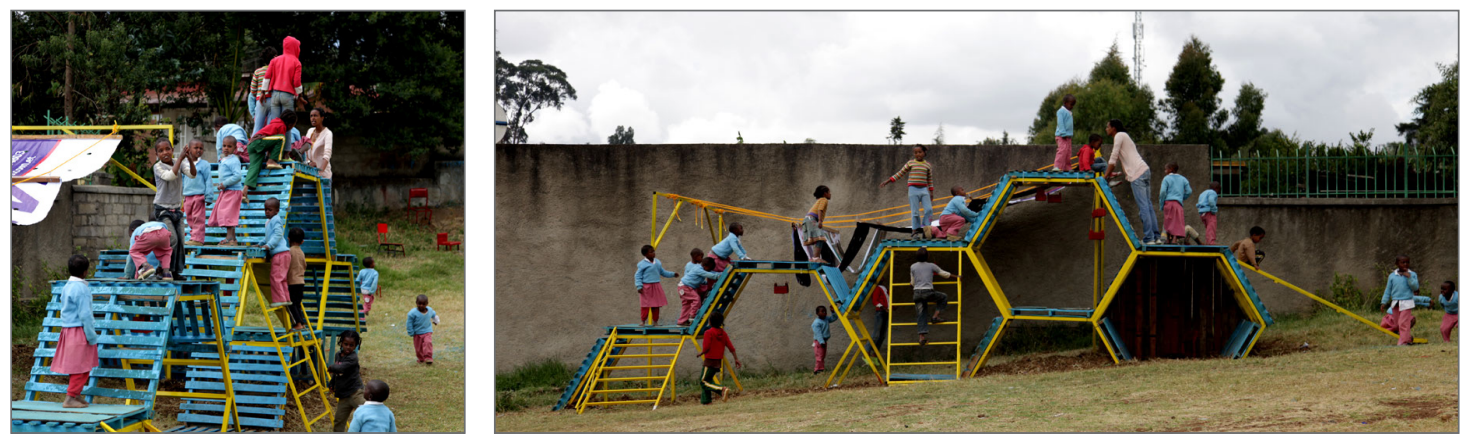

Fig. 6 y 7. Autoparque en Addis Abeba. La casa de los niños perdidos. Auto- / espacio público, 2012. Basurama. Addis Abeba, Etiopía. Chatarra. Lona publicitaria. Palés. Ropa. http://basurama.org/proyecto/autoparque-en-addis-abeba-la-casa-de-los-ninos-perdidos/

\section{Groundswell}

Centro de arte comunitario creado en Nueva York en 1996 para promover el arte colaborativo a través de la interacción y el activismo en la comunidad. Su organización está dirigida por artistas, activistas y educadores, que se reúnen para dar riqueza visual mediante la intervención en espacios urbanos, murales e instalaciones artísticas y los artistas trabajan en grupos reducidos.

El mural expuesto es del año 2012 y realizado mediante una colaboración entre entidades como: Centro de Justicia Comunitaria y el Proyecto de Granja Estudiantil de Brownsville. El Fondo Nacional para las artes, dio los medios para el proyecto: Transform / Restore: Brownsville, para embellecer paredes con murales pintados por jóvenes adultos en libertad condicional. 
Este proyecto se diseñó para jóvenes con un alto riesgo de exclusión social para realizar Piezas creativas permanentes y visibles en zonas conflictivas de Nueva York. Cuarenta jóvenes en libertad condicional crearan cinco proyectos de arte público de gran tamaño para ser visibles para los residentes del barrio, con la mayor concentración de viviendas públicas y una población importante de reclusos.

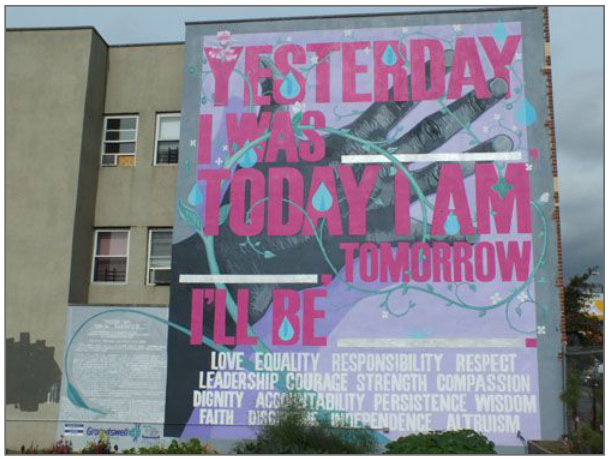

Boa Mistura
Fig. 8. Proyecto Mural Comunitario de Groundswell, 2013. Nueva York. Artista: Chris Soria Artista asistente: Jules Joseph www.arts.gov/national/our-town/grantee/2013/ groundswell-community-mural-project

El poblado de Khayelitsha es uno de los más grandes de Sudáfrica, con unos 400.000 habitantes, después de Soweto (Johannesburgo). Los materiales de las casas están realizados con chapas, cartones y maderas. Estas zonas habitadas por residentes rurales son los asentamientos que se ofrecían para los individuos que eran los "no blancos" durante el periodo del Apartheid. BoaMistura visito el centro Velokhaya, escuela de ciclismo y centro cultural que entrena a niños, inculcándoles valores humanos con la práctica del deporte; un lugar de encuentro ante las dificultades que encuentran en sus casas. Tras dialogar con su creador y los monitores, se pensó en una forma en la que pudieran colaborar pintando el mural y los contenedores. Este proceso fue determinante para Boa Mistura, una experiencia que modificaría su percepción acerca del arte urbano. Al observar que cuando las personas pintan y transforman el lugar en el que habitan, se reconocen, lo hacen suyo $\mathrm{y}$ tratan de mejorarlo.

La última intervención del colectivo se ha realizado en la Cañada Real (Madrid), su actividad es el predominio del color, así como de palabras y frases significativas sobre los muros pintados. Es un espacio que está determinado por fomentar un interés ecológico y de carácter etnográfico. Se trata de revalorizar y conservar las cañadas, tanto su diseño como su creación están 
suponiendo una importante implicación social con una difusión en los medios notable.
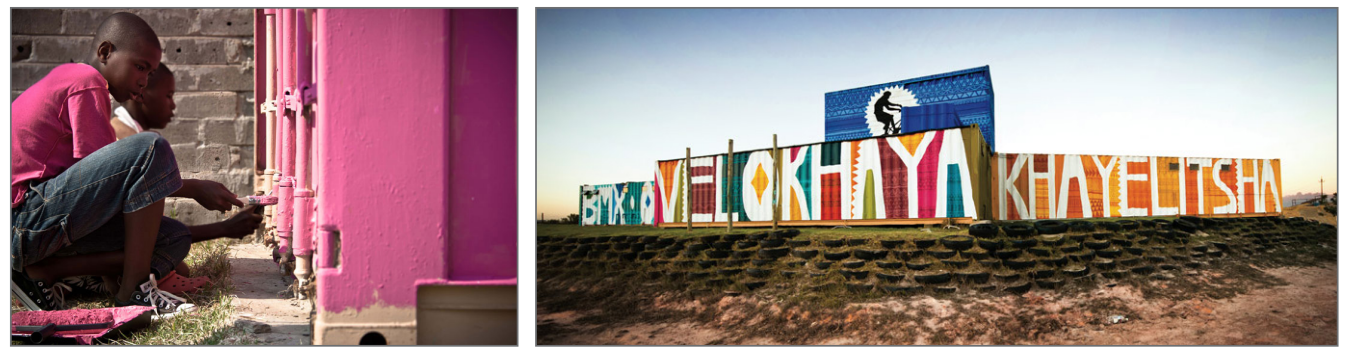

Fig. 9 y 10. Velokhaya. 2011. Ciudad del Cabo, Sudáfrica. Boa Mistura.

http://www.boamistura.com/\#/project/velokhaya

\section{Bosque de alimentos de Beacon}

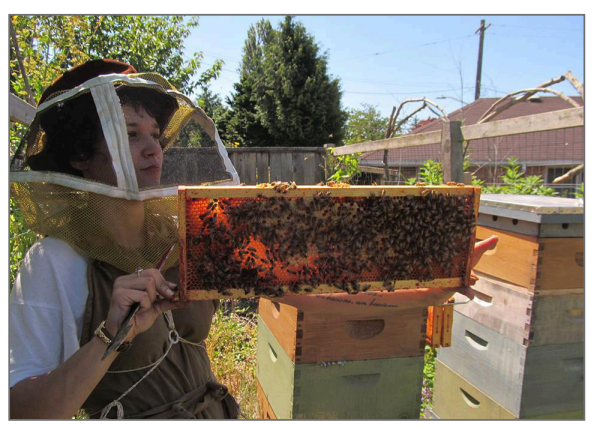

Fig.11. Beacon Food Forest, 2018. http://beaconfoodforest.org/
Espacio de producción de alimentos en Seattle, para cultivo de frutas y hortalizas en terreno público. Se creó con la ayuda de la comunidad de Beacon Hill, Harrison Design y Permaculture Now! Su objetivo es proporcionar alimentos saludables a miembros de la comunidad y generar un ambiente de cooperación e intercambio cultural. Presenta un área para niños, con actividades de juego y aprendizaje, una zona de bosque de nueces y un espacio de bayas. La iniciativa ha sido muy valorada y el contacto a través de los productos crea unos vínculos humanos que se mantienen.

\section{Candy Chang}

En su instalación artística participativa realizada en la ciudad finlandesa de Turku titulada Trayectoria profesional, fue una invitación a la artista para diseñar un proyecto de tipo comunitario y participativo. Su localización entre la universidad y las viviendas de los estudiantes era como un espacio para escribir. Se creó una frase de plantilla que se transfirió al asfalto con la frase: "Cuando yo era pequeño, quería ser un , ahora quiero ser un ", en este camino se proporcionan tizas para que la comunidad diera sus respuestas. 

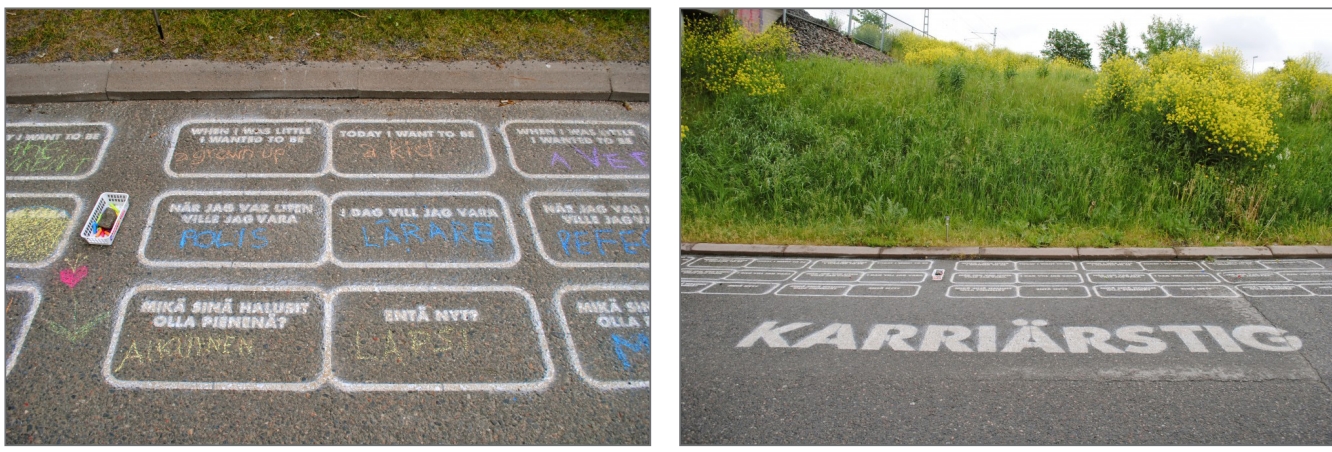

Fig. 12 y 13. Trajectoria profesional, 2011. Candy Chang. http://candychang.com/work/career-path/

\section{APLICACIÓN EN ESPACIOS DEL ARTE COMUNITARIO EN LA COMUNIDAD DE CANARIAS}

Dentro del mundo del arte, el arte comunitario es considerado como expresión artística importancia menor. Sarah Kent, en una exposición del 1976 organizada por la Tower Hamlets Arts Project en la galería London Whitechapel manifiesta su opinión acerca del arte comunitario y su relación con las galerias: "El arte comunitario y las muestras en galerías me parecen ideológicamente opuestas: el arte comunitario se justifica por la calidad de la participación de los participantes y no por el producto final. Presentarlos para su exhibición, por lo tanto, parece absurdo y sugiere ilusiones de grandeza inapropiadas para el propósito, la función y la postura política de las artes de la comunidad" (citada en Walker 2002, 134).

\subsection{Artistas 0 experiencias aplicadas a esta disciplina}

El arte comunitario en su diseño, planificación e implementación en canarias puede ser analizado desde una doble vertiente. Por un lado, el análisis de aquellos artistas que están comprometidos con enfatizar la identidad canaria y potenciar lugares para que no se propicie una degradación del territorio; éstos estarían más próximos al arte, su objetivo es valorar y difundir la identidad canaria. También se analizan instituciones educativas en las que a través fundamentalmente de murales se involucra a la comunidad educativa para su realización y promover valores educativos y de integración de las personas que la conforman. 


\section{Sabotaje al montaje}

Desde el año 1998 Sabotaje al Montaje ha creado proyectos y participado activamente en eventos, a nivel nacional e internacional. Su creador es licenciado en Bellas Artes por la Universidad de la Laguna en el año 2003. En la actualidad experimenta a partir del graffiti mural de amplias dimensiones, obteniendo un reconocimiento en el $1^{\circ}$ Concurso Internacional de artes urbanas Artaq festival París \& Berlín en el 2010. En esta obra de Puerto Santiago (Tenerife), se muestran las tradiciones más arraigadas de la isla, de aquellos que cultivaron la tierra, dignificando la identidad canaria desde sus raíces. La calidad de sus creaciones ha generado un interés y reconocimiento en el archipiélago canario.
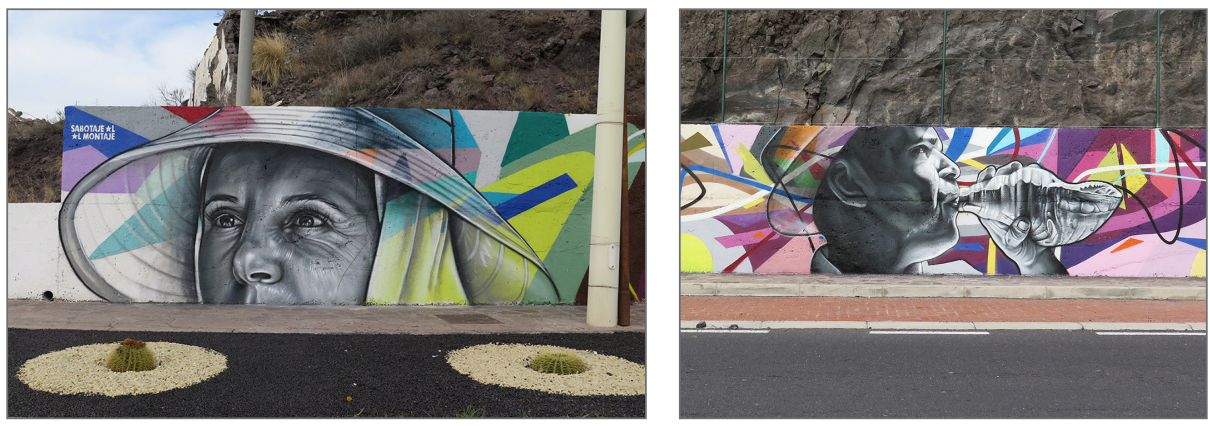

Fig. 14 y 15. Puerto Santiago Tenerife. Mural participativo/Ayuntamiento Santiago del Teide - Juventud 2015. http://www.sabotajealmontaje.com/

\section{Fernando Menis}

El municipio de La Oliva en Fuerteventura, ha fomentado el turismo desde siempre. Con anterioridad lo han hecho con planteamientos estandarizados, pero ahora se está propiciando a través de propuestas innovadoras. En este sentido, el referente es la isla de Lanzarote que, debido a las intervenciones de Cesar Manrique, existen espacios que han sido declarados como Bienes de Interés Cultural. Con este objetivo el actual alcalde del municipio de la Oliva realiza un encargo al arquitecto Fernando Menis, para efectuar el diseño de la iluminación de Navidad del 2017, con los objetivos siguientes:

- Planificar un diseño de calidad y adaptado culturalmente a La Oliva, tradicionalmente vinculada al mar y la pesca.

- Transformar la zona a través de la iluminación navideña en un centro de interés turístico.

- Construir una iluminación sostenible en relación al consumo energético y con materiales reciclados. 
En base a estas propuestas, Menis diseñó varias instalaciones con objetos reciclados y materiales abandonados por niños y turistas en hoteles: tablas de surf, juguetes, flotadores, etc. La decoración se creó entre los miembros del municipio y se ha difundido en revistas internacionales de Arte y Arquitectura.
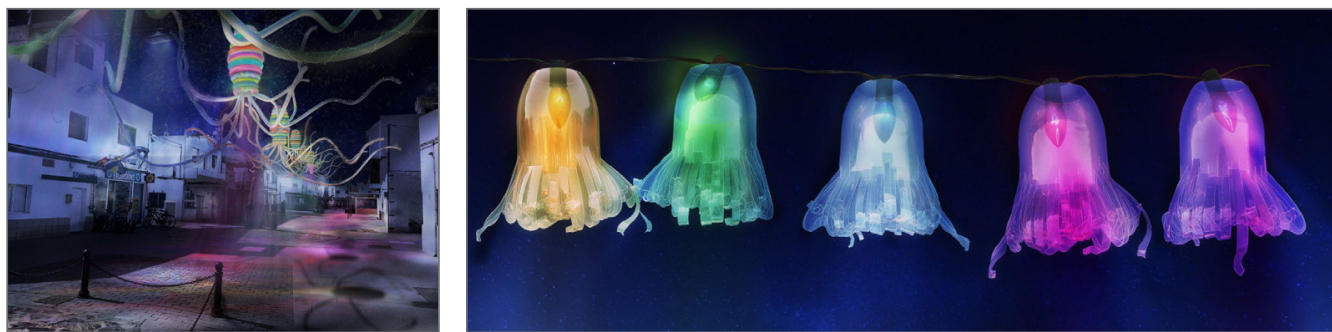

Fig. 16 y 17. Iluminación Navideña, 2017. Fernando Menis. Fuerteventura.

http://menis.es/portfolio/iluminacion-navidena-en-la-isla-de-fuerteventura-islas-canarias/

\subsection{LA CREACIÓN ARTÍSTICA COMUNITARIA EN CENTROS EDUCATIVOS CANARIOS}

El centro de la Aneja en La Laguna en el año 2013 impulsó una pintura mural con la Asociación de Madres y Padres de alumnos en el proyecto: "Pinta una estrella". Recibió una mención especial que fue reconocida en el XIV Concurso de Experiencias Educativas y organizado por la Confederación Española de Asociaciones de Madres y Padres de Alumnos. Para su realización sus miembros propusieron la ejecución de un gran mural en las paredes de la zona del exterior del colegio con participación de los niños. Para ello, pintaron un espacio en el que se representa un firmamento repleto de estrellas de formas diversas, colores y tamaños en las que figuran los nombres de los niños. El proyecto ha sido reconocido a nivel nacional y ha alcanzado su finalidad: representación de todos los niños del centro de cada curso.
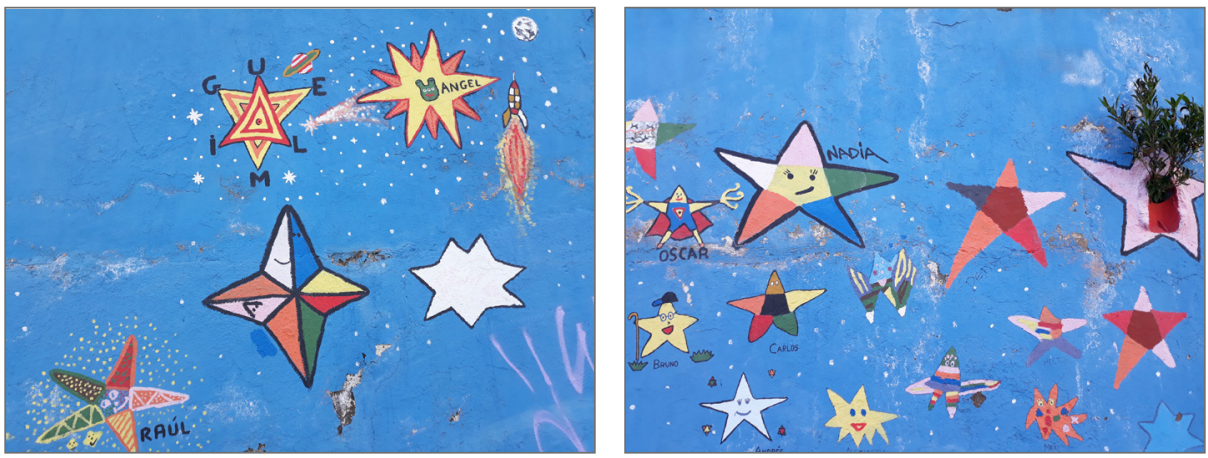

Fig.18 y 19. Pinta una estrella. Mural colaborativo. CEIP Aneja, 2013. La Laguna, Tenerife. Fotografías propias. 


\section{Lista de tareas de la comunidad}

- Identificar cómo podemos dividir nuestra comunidad en equipos.

- Asegurar que los equipos puedan comunicarse de manera clara y efectiva.

- Atraer a una amplia gama de colaboradores a la comunidad para que se involucren y contribuyan a los objetivos.

- Construir un ambiente propicio para nuestros objetivos más amplios.

- Defina el alcance de cada equipo y ayude a los miembros del equipo a comprender ese alcance.

- Comprender el alcance y el rango de colaboración entre nuestros equipos.

- Fomentar la diversidad y la oportunidad en la comunidad.

- Producir un Código de Conducta.

Tabla extraída del libro: The Art of Community. p. 43

\section{METODOLOGÍA EMPLEADA}

La metodología que se ha utilizado para investigar sobre el arte comunitario tiene una base teórica y de análisis, a través de la búsqueda de material sobre la materia en el campo del arte y de la educación. Además, se considera que se podría enfocar y ampliar este formato de investigación para una visión más práctica del ámbito de conocimiento artístico y la posible aplicación a través del aprendizaje basado en proyectos y aprendizaje basado en servicio a una comunidad.

Sin embargo, para la creación de propuestas artísticas de arte comunitario se estima oportuno que la metodología heurística sería la más idónea para implementar proyectos artísticos específicos con el empleo de unas reglas empíricas para alcanzar la solución. Para su utilización se deben incluir cinco fases a seguir: reconocer el problema específico, analizar y mostrarlo y desarrollar estrategias factibles; alcanzar la solución y evaluar los resultados de las actividades.

David Gauntlett, con formación como sociólogo, empleó elementos de Lego para manifestar la identidad y desarrolló el juego "Lego Serious Play": "En términos prácticos, Lego Serious Play es un concepto y metodología que ayuda a las organizaciones a tener reuniones más efectivas para resolver problemas estratégicos complejos (Gauntlett 2007, 129). 


\section{CONTRIBUCIÓN CIENTÍFICA DE LA INVESTIGACIÓN}

La aportación más relevante que se realiza a través de este artículo es visibilizar como el arte comunitario puede favorecer determinados espacios para valorar a través del arte la identidad de un lugar específico. Así como abrir el espectro no sólo al ámbito del arte, sino al de la educación, para que las instituciones educativas valoren esta forma de arte e involucren a la comunidad y desarrollen iniciativas en los espacios de los que disponen para su desarrollo e implementación.

El artista alemán Eberhard Bosslet ha realizado proyectos artísticos en las islas del Hierro, Tenerife y Lanzarote, desde una actitud de respeto al medio y cuidando que la intervención artística sea adecuada a las islas. Además, Fernando Menis creo una iluminación en la isla de Fuerteventura en la que no sólo se involucra a la comunidad isleña, sino que se mantiene la identidad del lugar en el que fue específicamente diseñado y a través de planteamientos y soluciones artísticas de reciclaje.

En relación a las acciones educativas existentes en la isla de Tenerife el Colegio Aguere situado en San Cristobal de La Laguna ha creado un huerto escolar en el que alumnado de infantil y primaria colabora de forma activa en el mismo para promover una enseñanza de respeto al medio y a los animales, pues es la riqueza de las islas. El mural del Colegio de la Aneja diseño un mural en que la comunidad educativa y alumnado de esta institución colaboraron en su diseño y creación para generar un espacio de identidad y respeto a esa zona de enseñanza.

Las acciones colaborativas realizadas en las islas pueden ser un ejemplo de lo que se puede llevar a cabo con una proyección pensada desde lo local con visión global. Es por esto, que los proyectos ambientales que promueve el gobierno del estado de Utah que se exponen en el proyecto de Servicios Sociales destinados a una comunidad joven Youth Service America $\left(\mathrm{YSA}^{2}\right)$, son manifestaciones de modelos artísticos con visión educativa que pueden ser implementados en las islas siempre valorando y respetando la identidad isleña que es lo que mantiene la calidad de vida de nuestro hábitat tan preciado. 


\section{CONCLUSIONES}

Aunque las actividades artísticas en comunidades se han realizado desde siempre y se conoce bien su poder de integración, como la realización de alfombras florales en las islas canarias o los jardines y patios surgidos en relación a motivaciones comunitarias, éstas no siempre promueven procesos de cambio o de justicia social. Por ello, nos referimos principalmente a comunidades de carácter periurbano sin una integración real o a comunidades discriminadas que fomentan una transformación social.

Se ha iniciado un movimiento de innovación en el que las asociaciones colaboran con artistas para coproducir festivales en el ámbito de la cultura que exponen problemáticas que impliquen a la sociedad. Este formato artístico se ha incrementado en la educación artística, debido a que se crean nuevos discursos en arte contemporáneo y conforman la identidad del individuo en un espacio de comunidad; por lo tanto, es un modo idóneo para enseñar arte en centros escolares, pues no sólo se consideran aspectos estéticos, sino que se valora el proceso artístico y aspectos de justicia social.

Debido a que el ámbito artístico actual se ha ampliado en base a conceptos innovadores y ciertas prácticas han sido desarrolladas por organizaciones que involucran a distintas disciplinas, es preciso que los valores sobre los que se construya la acción artística se fundamenten en una ética respetuosa con la comunidad, una formación interdisciplinaria en arte que se materialice en prácticas que promueven los valores identitarios del grupo.

\section{Referencias}

Bacon, Jono. 2012. The Art of Community: Building the New Age of Participation. Sebastopol CA: O’Reilly

Barragán Rodríguez, José María \& Ascensión Moreno González. 2004. "Experiencia artística y producción cultura, ámbitos para la intervención socieducativa”. Educación Social 28: $19-40$

Bishop, Claire. 2004. "Antagonism and relational aesthetics". October 110 (Fall): 51-79. https:// www.mitpressjournals.org/doi/pdf/10.1162/0162287042379810

— . 2006. "The social turn: Collaboration and its discontents". Art Forum 6. https://www. artforum.com/inprint/id=10274

Bourriaud, Nicolas. 1994. Relational aesthetics. Translated by Simon Pleasance \& Fronza Woods with the participation of Mathieu Copeland. Dijon: Du Reel

— . 2009. Altermodern: Tate Triennial 2009. London: Tate 
Burgin, Victor. 1986. The end of art theory: Criticism and postmodernity. Atlantic Highlands NJ: Humanities Press International

Chonody, Jill M., ed. 2014. Community art: Creative approaches to practice. Champaign IL: Common Gound

Congdon, Kristin G. 2004. Community art in action. Worcester, MA: Davis

Coutts, Glen \& Jokela Timo. 2008. Art, community and enviroment: Educational perspectives. Bristol: Intellect Books

Crehan, Kate. 2011. Community art: An anthropological perspective. London: Berg

Crespo Martin, Bibiana. 2016. "Arte participativo en el espacio público: Proposiciones metodológicas acerca de algunos de sus preceptos". On the W@terfront 45(2) http:/l revistes.ub.edu/index.php/waterfront/article/view/18678

Cunha Leal, Joana. 2010. "On the strange place of public art in contemporary art theory". On the W@terfront 16:35-52._http://revistes.ub.edu/index.php/waterfront/article/view/18711

Delgado Ruiz, Manuel. 2007. Sociedades movedizas: Pasos hacia una antropología de las calles. Barcelona: Anagrama

Efland, Arthur D., Kerry Freedman \& Patricia Stuhr. 2003. La educación en el arte posmoderno. Traducción de Lucas Vermal. Barcelona: Paidós

Eisner, Elliot. 2004. El arte y la creación de la mente: El papel de las artes visuales en la transformación de la conciencia. Traducción de Genís Sánchez Barberán. Barcelona: Paidós

Foster, Hal. 2006. "Chat rooms". En Participation, edited by Claire Bishop. London: Whitechapel Gallery

Gauntlett, David. 2007. Creative explorations: New approaches to identities and audiences. London: Routledge

Haacke, Hans. 1971. "An interview with Hans Haacke" by Jeanne Siegel. Arts Magazine 45(7): 21

Hardy, Sherrill. 2017. Environmental sculptures: Sculpture installations. Victoria BC: FriesenPress

Harvey, David. 1989. The condition of postmodernity: An enquiry into the origins of cultural change. Oxford: Basil Blackwell

Jiménez Sequeiros, María. 2015. "Arte de contexto y acción social. Prácticas artísticas colaborativas en el contexto social español a comienzos del siglo XX". Tesis Univ. Barcelona. http://diposit.ub.edu/dspace/handle/2445/104272

Kelly, Owen. 1984. Community, art and the state: Storming the citadels. London: Comedia

Kester, Grant H. 2004. Conversation pieces: Community and communication in modern art. Berkeley CA: University of California

Krensky, Beth \& Seana Lowe Steffen. 2009. Engaging classrooms and communities through art: $A$ guide to designing and implementing community-based art education. Lanham MD: Altamira

Lacy, Suzanne. 1995. Mapping the terrain: New genre public art. Seattle WA: Bay 
Lind, Maria. 2007. "The collaborative turn". En Taking the matter into common hands: On contemporary art and collaborative practices, edited by Johannan Billing, Maria Lind \& Lars Nilsson. London: Black Dog

Lippard, Lucy R. 2004. Seis años: La desmaterialización del objeto artístico de 1966 a 1972. Traducción, $M^{a}$ Luz Rodríguez Olivares. Madrid: Akal

MACBA. 2006. "MACBA any 10, moment 0". En Recull de textos de l'Agenda informativa del MACBA Ag 2004-2006, 16-17. http://macba.es/uploads/20140211/QP_05_Agenda_cat.pdf

O’Neill, Paul \& Mick Wilson, eds. 2009. Curating and the educational turn. London: Open

Powazek, Derek M. 2001. Design for community: The art of connecting real people in virtual places. San Indianapolis IN: New Riders

Ribalta Delgado, Jorge. 2004. "Contrapúblicos: Mediación y construcción de públicos”. Republicart.net. http://republicart.net/disc/institution/ribalta01_es.htm

Ricart Ulldemolins, Núria. 2009. “Cartografies de La Mina: Art, espai públic, participació ciutadana”. Tesis Univ. Barcelona. http://hdl.handle.net/10803/1549

Rose, Gillian. 2012. Visual methodologies: An introduction to interpreting visual materials. London: Sage

Smith, Hazel \& Roger T. Dean. 2009. Practice-led research, research-led practice in the creative arts. Edinburgh: Edinburgh University

Suderburg, Erika. 2000. Space, site, intervention: Situating installation art. Minneapolis MN: University of Minnesota

Ukeles, Mierle Laderman. 1969. "Manifesto for maintenance art 1969!: Proposal for an exhibition 'care'”. http://www.queensmuseum.org/wp-content/uploads/2016/04/Ukeles MANIFESTO.pdf

Veal, Clare. 2014. "Bringing the land foundation back to Earth: A new model for the critical analysis of relational art" Journal of Aesthetics \& Culture 6. http://dx.doi.org/10.3402/jac. v6.23701

Walker, John Albert. 2002. Left shift: Radical art in 1970s Britain. London: I. B. Taurus

\section{Notas}

${ }^{1}$ http://assemblestudio.co.uk

2 YSA (Youth Service America). 2013. 55-Environmental service-projects. Excerpted and adapted from Take Care of Oregon Days: 150 Service-Learning Project Ideas (SOLV 2008). https://heritage.utah.gov/wp-content/uploads/55-Environmental-service-projects. pdf?x15791z 